\title{
Four-loop results for the cusp anomalous dimension
}

\author{
R. Brüser ${ }^{a}$, A. G. Grozinn ${ }^{* b, c}$, J. M. Henn ${ }^{a, d}$ and M. Stahlhofen ${ }^{a}$ \\ a PRISMA Cluster of Excellence, Johannes Gutenberg-Universität, 55128 Mainz, Germany \\ ${ }^{b}$ Budker Institute of Nuclear Physics, Novosibirsk 630090, Russia \\ ${ }^{c}$ Novosibirsk State University, Novosibirsk 630090, Russia \\ ${ }^{d}$ Max-Planck-Institut für Physik, Werner-Heisenberg-Institut, 80805 München, Germany \\ E-mail: brueser@uni-mainz.de, A.G.Grozin@inp.nsk.su, \\ henneuni-mainz.de and mastahlheuni-mainz.de
}

\begin{abstract}
We review the current status of calculations of the HQET field anomalous dimension and the cusp anomalous dimension. In particular, we give the results at 4 loops for the quartic Casimir contribution, and for the full QED case, up to $\varphi^{6}$ in the small angle expansion. Furthermore, we discuss the leading terms in the anti-parallel lines limit at four loops.
\end{abstract}

Loops and Legs in Quantum Field Theory (LL2018)

29 April 2018 - 04 May 2018

St. Goar, Germany

${ }^{*}$ Speaker. 


\section{Introduction}

We review the current status of calculations of 2 QCD quantities: the HQET field anomalous dimension $\gamma_{h}$ and the cusp anomalous dimension $\Gamma_{\text {cusp }}(\varphi)$. Due to non-abelian exponentiation, they have only a subset of all possible color structures. At small angles $\Gamma_{\text {cusp }}(\varphi)$ is a regular series in $\varphi^{2}$; at large angles $\Gamma_{\text {cusp }}(\varphi)=K \varphi+\mathscr{O}\left(\varphi^{0}\right)$, where $K$ is the light-like cusp anomalous dimension. These quantities are known at 3 loops: $[1,2]$ and $[3,4]$. The status of 4-loop calculations is summarized in Table 1.

\begin{tabular}{l|l|l|l|l}
\hline & $\gamma_{h}$ & $\Gamma_{\text {cusp }}(\varphi)$ & $\varphi \ll 1$ & $\varphi \gg 1$ \\
\hline$C_{F}\left(T_{F} n_{l}\right)^{3}$ & {$[5]$} & {$[6]$} & & \\
\hline$C_{F}^{2}\left(T_{F} n_{l}\right)^{2}$ & {$[4,7]$} & {$[4,7]$} & & \\
$C_{F} C_{A}\left(T_{F} n_{l}\right)^{2}$ & {$[8,9]$} & & {$[9]$} & {$[10,11]$} \\
\hline$C_{F}^{3} T_{F} n_{l}$ & {$[12]$} & {$[12]$} & & \\
$d_{F F} n_{l}$ & {$[13]$} & & {$[13]$} & {$[14,15] *$} \\
$C_{F}^{2} C_{A} T_{F} n_{l}$ & {$[8] *$} & & & {$[14] *$} \\
$C_{F} C_{A}^{2} T_{F} n_{l}$ & {$[8] *$} & & & {$[14] *$} \\
$n_{l}^{1}, N_{c} \rightarrow \infty$ & & & & {$[10,14]$} \\
\hline$C_{F} C_{A}^{3}$ & {$[8] *$} & & & {$[14] *$} \\
$d_{F A}$ & {$[8] *$} & & & {$[14,15] *$} \\
$n_{l}^{0}, N_{c} \rightarrow \infty$ & & & & {$[16,14]$} \\
\hline $\mathrm{QED}$ & {$[12]$} & & {$[12]$} &
\end{tabular}

Table 1: 4-loop contributions to $\gamma_{h}$ and $\Gamma_{\text {cusp }}(\varphi)$. The sign $*$ means that the contribution is only known numerically.

The calculation of the $C_{F} C_{A}\left(T_{F} n_{l}\right)^{2}$ structure in $\Gamma_{\text {cusp }}(\varphi)$ at $\varphi \ll 1$ is in progress [9]. The $C_{F}^{3} T_{F} n_{l}$ structure is discussed in Sect. 2 [12], and the $d_{F F} n_{l}$ structure (where $d_{F F}=d_{F}^{a b c d} d_{F}^{a b c d} / N_{F}$ ) - in Sect. 3 [13]. Not much is known about the $C_{F} C_{A}^{3}$ structure; when the Euclidean $\varphi$ is $\pi-\delta$, $\delta \rightarrow 0$, it has a $\log (\delta) / \delta$ term (Sect. 4 [17]) (calculation of the non-logarithmic $1 / \delta$ term is much more difficult and not yet done). form

It has been conjectured in $[3,4]$ that the cusp anomalous dimension can be represented in the

$$
\Gamma_{\text {cusp }}(\varphi)=C_{F} \frac{a}{\pi}\left[\Omega(\varphi)+C_{A} \Omega_{A}(\varphi) \frac{a}{\pi}+C_{A}^{2} \Omega_{A A}(\varphi)\left(\frac{a}{\pi}\right)^{2}\right]+\mathscr{O}\left(a^{4}\right)
$$

containing no $n_{l}$, via the effective coupling

$$
\begin{aligned}
\frac{a}{\pi}= & \frac{\alpha_{s}}{\pi}+\left(C_{A} B_{A}+T_{F} n_{l} B_{l}\right)\left(\frac{\alpha_{s}}{\pi}\right)^{2}+\left(C_{A}^{2} B_{A A}+C_{F} T_{F} n_{l} B_{F l}+C_{A} T_{F} n_{l} B_{A l}+\left(T_{F} n_{l}\right)^{2} B_{l l}\right)\left(\frac{\alpha_{s}}{\pi}\right)^{3} \\
& +\mathscr{O}\left(\alpha_{s}^{4}\right)
\end{aligned}
$$

which is determined from the condition that at $\varphi \rightarrow \infty$ the $\mathscr{O}(\varphi)$ asymptotics is given by the first term in (1.1). This is true up to 3 loops. For example, the 3-loop $C_{F} C_{A} T_{F} n_{l}$ term in $\Gamma_{\text {cusp }}(\varphi)$ (a typical diagram is shown in Fig. 1) is a combination of 2- and 1-loop terms:

$$
\Gamma_{\text {cusp }}(\varphi)=\cdots+C_{F} C_{A} T_{F} n_{l}\left[B_{A l} \Omega(\varphi)+2 B_{l} \Omega_{A}(\varphi)\right]\left(\frac{\alpha_{s}}{\pi}\right)^{3}+\cdots
$$


This conjecture has been disproved for a quartic Casimir color structure [13] (Sect. 3). Remarkably, numerically the conjectured formula is very close to the exact one, cf. Sect. 3.

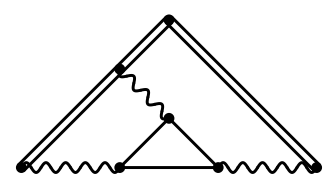

Figure 1: A diagram for the 3-loop $C_{F} C_{A} T_{F} n_{l}$ term in $\Gamma_{\text {cusp }}(\varphi)$.

2. $C_{F}^{3} T_{F} n_{l}$

This is a QED problem. Due to exponentiation, the coordinate-space propagator of the BlochNordsieck field (i.e. the straight Wilson line $W$ ) is

$$
W=\exp \left(\sum w_{i}\right)
$$

where $w_{i}$ are single-web diagrams. Due to $C$ parity conservation in QED, webs have even numbers of legs (Fig. 2). Webs with 4 legs (Fig. 2b) first appear at 4 loops (Sect. 3). All contributions to $\log W(2.1)$ are gauge invariant except the 1-loop one, because proper vertex functions with any numbers of photon legs are gauge invariant and transverse with respect to each photon leg due to the QED Ward identities.

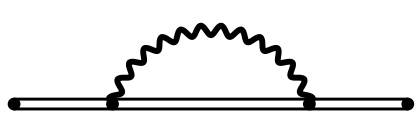

a

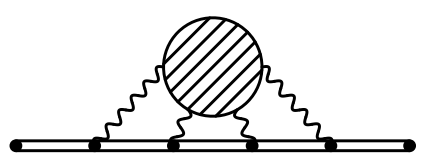

$\mathrm{b}$

Figure 2: Webs: (a) 2-leg (the thick line is the full photon propagator); (b) 4-leg (the blob is the sum of connected diagrams).

In Landau gauge we obtain

$$
\gamma_{h}=\frac{\alpha}{4 \pi}\left[-6+n_{l} \sum_{L=1}^{\infty}\left(-6 \bar{\Pi}_{L}+2 \bar{\beta}_{L}\right)\left(\frac{\alpha}{4 \pi}\right)^{L}\right]+\left(n_{l}^{>1} \text { terms }\right)+\left(w_{>2 \text { legs }} \text { terms }\right),
$$

where the photon self energy is

$$
\Pi_{L}=\left(\frac{\bar{\beta}_{L}}{L \varepsilon}+\bar{\Pi}_{L}\right) n_{l}+\left(n_{l}^{>1} \text { terms }\right), \quad \beta_{L}=\bar{\beta}_{L} n_{l}+\left(n_{l}^{>1} \text { terms }\right)
$$

( $\beta_{L}$ is the $L$-loop $\beta$ function coefficient). Substituting $\bar{\Pi}_{L}$ [18], restoring color structures and inserting the 1-loop gauge dependence, we obtain up to 5 loops [12]

$$
\gamma_{h}=2(a-3) C_{F} \frac{\alpha_{s}}{4 \pi}+T_{F} n_{l} C_{F}\left(\frac{\alpha_{s}}{4 \pi}\right)^{2}\left[\frac{32}{3}-6\left(16 \zeta_{3}-17\right) C_{F} \frac{\alpha_{s}}{4 \pi}\right.
$$




$$
\begin{aligned}
& +\frac{16}{3}\left(180 \zeta_{5}-111 \zeta_{3}-35\right)\left(C_{F} \frac{\alpha_{s}}{4 \pi}\right)^{2} \\
& \left.-6\left(2240 \zeta_{7}-1960 \zeta_{5}-104 \zeta_{3}-5\right)\left(C_{F} \frac{\alpha_{s}}{4 \pi}\right)^{3}+\mathscr{O}\left(\alpha_{s}^{4}\right)\right]+ \text { (other color structures) }
\end{aligned}
$$

Now we consider the cusped Wilson line $W(\varphi)$ from $x=-v t$ to 0 and then to $x^{\prime}=v^{\prime} t$ :

$$
\log \frac{W(\varphi)}{W(0)}=\sum\left(w_{i}(\varphi)-w_{i}(0)\right)
$$

Diagrams in which all photon vertices are to the left (or to the right) of the cusp cancel in $w_{i}(\varphi)-$ $w_{i}(0)$. The remaining 2-leg webs are represented by Fig. 3. At 4 loops 4-leg webs appear (Sect. 3).

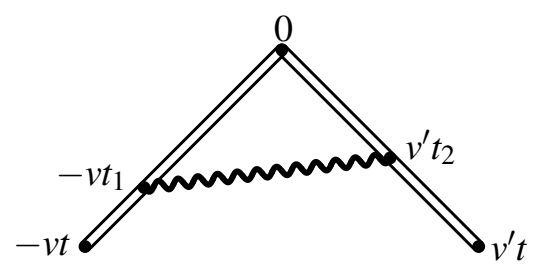

Figure 3: Cusp: the 2-leg webs (the thick line is the full photon propagator).

The $L$-loop $n_{l}^{1}$ contribution is proportional to

$$
\int_{0}^{t} d t_{1} \int_{0}^{t} d t_{2} v_{\mu} v_{v}^{\prime} \bar{D}_{L-1}^{\mu v}\left(v t_{1}+v^{\prime} t_{2}\right)
$$

where $\bar{D}_{L}^{\mu v}$ is the $n_{l}^{1}$ term in the $L$-loop photon propagator. Calculating the integral we obtain

$$
\Gamma_{\text {cusp }}(\varphi)=4(\varphi \operatorname{coth} \varphi-1) \frac{\alpha}{4 \pi}\left[1+n_{l} \sum_{L=1}^{\infty} \bar{\Pi}_{L}\left(\frac{\alpha}{4 \pi}\right)^{L}\right]+\left(n_{l}^{>1} \text { terms }\right)+\left(w_{>2} \text { legs terms }\right) .
$$

The QCD result up to 5 loops is [12]

$$
\begin{aligned}
& \Gamma_{\text {cusp }}(\varphi)=4(\varphi \operatorname{coth} \varphi-1) C_{F} \frac{\alpha_{s}}{4 \pi}\left\{1+T_{F} n_{l} \frac{\alpha_{s}}{4 \pi}\left[-\frac{20}{9}+\left(16 \zeta_{3}-\frac{55}{3}\right) C_{F} \frac{\alpha_{s}}{4 \pi}\right.\right. \\
& -2\left(80 \zeta_{5}-\frac{148}{3} \zeta_{3}-\frac{143}{9}\right)\left(C_{F} \frac{\alpha_{s}}{4 \pi}\right)^{2} \\
& \left.\left.+\left(2240 \zeta_{7}-1960 \zeta_{5}-104 \zeta_{3}+\frac{31}{3}\right)\left(C_{F} \frac{\alpha_{s}}{4 \pi}\right)^{3}+\mathscr{O}\left(\alpha_{s}^{4}\right)\right]\right\}+ \text { (other color structures) }
\end{aligned}
$$

3. $d_{F F} n_{l}$

Casimir scaling holds for $\gamma_{h}$ and $\Gamma_{\text {cusp }}(\varphi)$ up to 3 loops. At 4 loops quartic Casimir color structures $d_{R F} n_{l}$ and $d_{R A}$ appear, where $d_{R R^{\prime}}=d_{R}^{a b c d} d_{R^{\prime}}^{a b c d} / N_{R}, d_{R}^{a b c d}=\operatorname{Tr} t_{R}^{(a} t_{R}^{b} t_{R}^{c} t_{R}^{d)}, N_{R}=\operatorname{Tr} \mathbf{1}_{R}$. They cannot be represented as the quadratic Casimirs $C_{R}$ times a universal constant. Therefore, Casimir scaling breaks at 4 loops, unless by some miracle the coefficients of both quartic Casimirs identically vanish. But they don't vanish: in $\Gamma_{\text {cusp }}$ at Euclidean angle $\varphi_{E} \rightarrow \pi$ they are given by the 
corresponding coefficients in the 3-loop static potential, which are known [19] and non-zero. So, Casimir scaling breaks at 4 loops, as expected. This breaking has been shown not to vanish in other regions of $\varphi$, too: at Minkowsky angles $\varphi_{M} \gg 1$ (in $\mathscr{N}=4$ SYM $[20,21]$ and in QCD $[14,15]$ ) and at $\varphi \ll 1[13]$.

The $d_{F F} n_{l}$ contribution to the HQET self energy is given by 3 different diagrams (Fig. 4). It is gauge invariant due to QED Ward identities. We reduce these diagrams at residual energy $\omega<0$ to master integrals, and obtain $\varepsilon$ expansions of non-trivial master integrals using HYPERINT [22]. The result is [13]

$$
\left.\gamma_{h}\right|_{d_{F F} n_{l}}=-d_{F F} n_{l}\left(\frac{\alpha_{s}}{\pi}\right)^{4}\left(\frac{5}{4} \zeta_{5}-\frac{2}{3} \pi^{2} \zeta_{3}-\zeta_{3}+\frac{2}{3} \pi^{2}\right)
$$
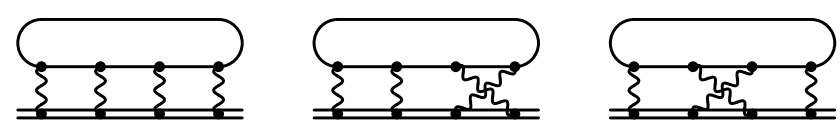

Figure 4: The $d_{F F} n_{l}$ contribution to the HQET self energy (symmetric diagrams implied).

We have also calculated the vertex at the residual energies of its legs $\omega_{1}=\omega_{2}$ expanded in $\varphi$. It is given by 6 different diagrams (Fig. 5); of course, it reduces to the same master integrals. The result is [13] (the $\varphi^{6}$ term is new)

$$
\begin{aligned}
& \left.\Gamma_{\text {cusp }}(\varphi)\right|_{d_{F F} n_{l}}=d_{F F} n_{l}\left(\frac{\alpha_{s}}{\pi}\right)^{4} \frac{\varphi^{2}}{9}\left[\pi^{2}\left(-4 \zeta_{3}+\frac{5}{12} \pi^{2}+\frac{5}{6}\right)\right. \\
& +\varphi^{2}\left(-4 \zeta_{5}-\frac{16}{75} \pi^{2} \zeta_{3}+\frac{71}{25} \zeta_{3}+\frac{49}{900} \pi^{4}-\frac{157}{900} \pi^{2}-\frac{23}{100}\right) \\
& \left.+\varphi^{4}\left(-\frac{64}{147} \zeta_{5}-\frac{32}{1225} \pi^{2} \zeta_{3}+\frac{983}{3675} \zeta_{3}+\frac{421}{66150} \pi^{4}-\frac{1333}{66150} \pi^{2}+\frac{797}{29400}\right)+\mathscr{O}\left(\varphi^{6}\right)\right] \\
& =d_{F F} n_{l}\left(\frac{\alpha_{s}}{\pi}\right)^{4} \varphi^{2}\left(0.150721+0.00965191 \varphi^{2}+0.000925974 \varphi^{4}+\mathscr{O}\left(\varphi^{6}\right)\right) .
\end{aligned}
$$
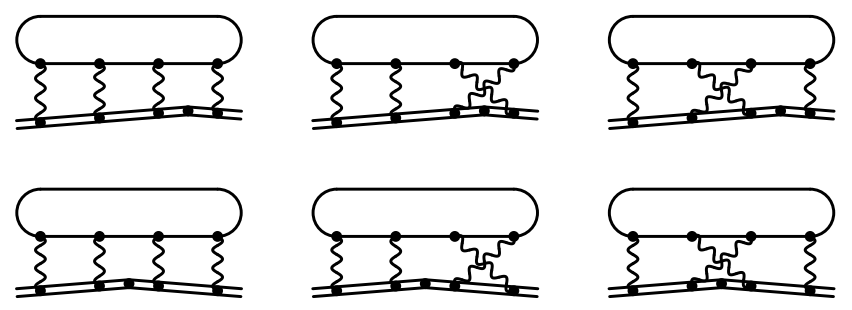

Figure 5: The $d_{F F} n_{l}$ contribution to the vertex (symmetric diagrams implied).

The conjecture from $[3,4]$ predicts

$$
\Gamma_{\text {cusp }}(\varphi)=C_{F} \Omega(\varphi) \frac{a}{\pi}+\cdots=\cdots+d_{F F} n_{l} B \Omega(\varphi)\left(\frac{\alpha_{s}}{\pi}\right)^{4}+\cdots, \quad \frac{a}{\pi}=\cdots+\frac{d_{F F} n_{l}}{C_{F}} B\left(\frac{\alpha_{s}}{\pi}\right)^{4}+\cdots
$$


The normalization factor $B$ can be found from the limit of euclidean $\varphi=\pi-\delta, \delta \rightarrow 0$, where the 4-loop $\Gamma_{\text {cusp }}(\varphi)$ is related to the 3-loop quark-antiquark potential which is known [23]. This gives the prediction

$$
\begin{aligned}
& \left.\Gamma_{\text {cusp }}(\varphi)\right|_{d_{F F} n_{l}} ^{\text {conj }}=d_{F F} n_{l}\left(\frac{\alpha_{s}}{\pi}\right)^{4} \frac{\varphi^{2}}{192}\left(1+\frac{\varphi^{2}}{15}+\frac{2}{315} \varphi^{4}+\mathscr{O}\left(\varphi^{6}\right)\right)\left(16 \pi^{4} \log ^{2} 2-336 \pi^{2} \zeta_{3} \log 2\right. \\
& \left.-\frac{16}{3} \pi^{4} \log 2-32 \pi^{2} \log 2+\frac{488}{3} \pi^{2} \zeta_{3}-\frac{5}{3} \pi^{6}+\frac{92}{3} \pi^{4}-\frac{632}{9} \pi^{2}\right) \\
& =d_{F F} n_{l}\left(\frac{\alpha_{s}}{\pi}\right)^{4} \varphi^{2}\left(0.14801+0.00986736 \varphi^{2}+0.000939748 \varphi^{4}+\mathscr{O}\left(\varphi^{6}\right)\right)
\end{aligned}
$$

So, the conjecture has been disproved. Curiously, the numerical values (3.4) of the coefficients predicted by the conjecture are quite close to the exact ones (3.2).

Adding (2.4), (3.1) and the known contributions with higher powers of $n_{l}$, we obtain the anomalous dimension of the Bloch-Nordsieck field in QED up to 4 loops, completely analytically. Adding (2.8), (3.2) and the known contributions with higher powers of $n_{l}$, we obtain the QED cusp anomalous dimension expanded up to $\varphi^{6}$.

\section{4. $\Gamma_{\operatorname{cusp}}(\pi-\delta)$}

This Section is based on work in progress [17]. In Euclidean space the angle $\varphi$ varies from 0 to $\pi$. When $\varphi=\pi-\delta, \delta \rightarrow 0$, the two world lines forming the cusp come together. At 2 loops $\Gamma_{\text {cusp }}(\pi-\delta) \sim 1 / \delta$, and the coefficient is related to the 1-loop quark-antiquark potential $V(r)$ [24]. This is explained by conformal symmetry; in QCD it is broken by the $\beta$ function, and at 3 loops this relation is broken by an extra term proportional to $\beta_{0}$ [3]. At 4 loops a new $\log (\delta) / \delta$ term appears (if no resummation is done). It is similar to the 3-loop $\log (\mu r)$ term in the static quark-antiquark potential $[25,26]$.

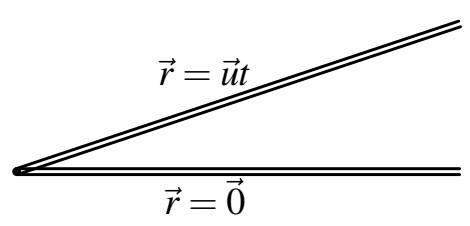

Figure 6: The Wilson line describing production of a heavy quark-antiquark pair with a small relative velocity $\vec{u}$.

Let's consider the cusped Wilson line in Minkowski space (Fig. 6). It is formed by the static quark and antiquark world lines $\vec{r}=0$ and $\vec{r}=\vec{u} t$, where $\vec{u}$ is the small relative velocity $(u=|\vec{u}| \ll 1)$. At the end of calculation we'll analytically continue the result to Euclidean space $(u=i \delta)$. We neglect all terms suppressed by powers of $u$. It is convenient to use Coulomb gauge. The static quark and antiquark interact by exchanging instantaneous Coulomb gluons:

$$
V(\vec{q})=-C_{F} \frac{g_{0}^{2}}{\vec{q}^{2}}, \quad V(\vec{r})=-C_{F} \kappa_{0} \frac{g_{0}^{2}}{4 \pi} \frac{1}{r^{1-2 \varepsilon}}
$$




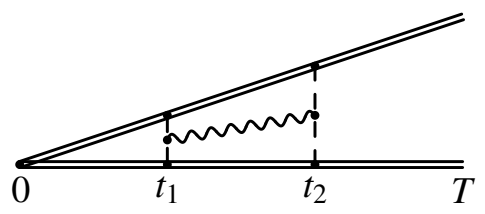

Figure 7: The first transverse-gluon contribution.

(the power of $r$ is obvious from dimensions counting). Here and below $\kappa_{i}=1+\mathscr{O}(\varepsilon)$ are some normalization factors (we don't need their exact form).

Transverse gluons interact only with Coulomb ones, but not with static quarks. The first transverse-gluon contribution is shown in Fig. 7. Here $T$ is an infrared cutoff. We use the method of regions to analyze this contribution. In the ultrasoft region $t_{1} \sim t_{2} \sim t_{2}-t_{1}$; Coulomb gluons characteristic momentum is $q \sim 1 /\left(u t_{1,2}\right)$, and the transverse gluon characteristic momentum is $k \sim 1 / t_{1,2} \ll q$. In the soft region $t_{2}-t_{1} \sim u t_{1,2}$, and $k \sim 1 /\left(t_{2}-t_{1}\right) \sim q$. To determine the coefficient of the logarithm in the $1 / \delta$ term in $\Gamma_{\text {cusp }}$, it turns out to be sufficient to consider the ultrasoft region [17]. Neglecting $k$ in the 3 -gluon vertex, we obtain in momentum and coordinate spaces

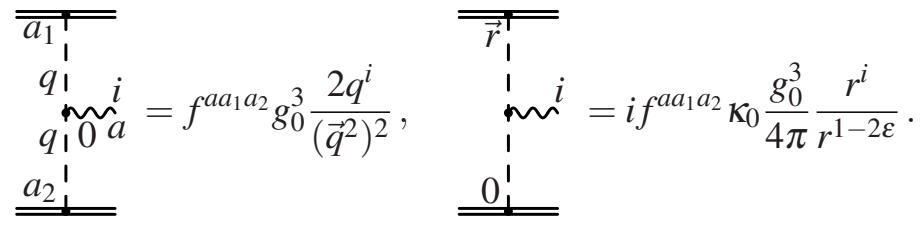

The ratio of the Wilson line (Fig. 7) to the one without the transverse-gluon correction is $1+R_{\mathrm{us}}+R_{\mathrm{soft}}$. The ultrasoft contribution is

$$
R_{\mathrm{us}}=\int_{0}^{T} d t_{2} \int_{0}^{t_{2}} d t_{1} K\left(t_{1}, t_{2}\right)
$$

where

$$
K\left(t_{1}, t_{2}\right)=\frac{1}{4} C_{F} C_{A}^{2} \kappa_{0}^{2} \frac{g_{0}^{6}}{(4 \pi)^{2}} \frac{r_{1}^{i}}{r_{1}^{1-2 \varepsilon}} \frac{r_{2}^{j}}{r_{2}^{1-2 \varepsilon}} D^{i j}\left(v\left(t_{2}-t_{1}\right)\right) \exp \left[-i \int_{t_{1}}^{t_{2}} d t \Delta V(u t)\right]
$$

$\left(v=(1, \overrightarrow{0})\right.$ is the 4 -velocity of our small dipole). During the time interval between $t_{1}$ and $t_{2}$, the static quark-antiquark pair is in the adjoint color state instead of the singlet one, and their leadingorder interaction potential $V_{o}(r)$ is obtained from the expression for the singlet potential $V(r)(4.1)$ by replacing the color factor $C_{F}$ with $C_{F}-C_{A} / 2$. Therefore, we get the integral of $\Delta V(r)=V_{o}(r)-$ $V(r)$. The characteristic sizes of the regions of the transverse gluon emission and absorption are $\sim u t_{1,2}$; we neglect them, so that this gluon propagates between the points $v t_{1}$ and $v t_{2}$ :

$$
D^{i j}(v t)=8(i / 2)^{2 \varepsilon} \frac{\Gamma(2-\varepsilon)}{3-2 \varepsilon} \frac{t^{-2+2 \varepsilon}}{(4 \pi)^{2-\varepsilon}} \delta^{i j}
$$

We obtain

$$
K\left(t_{1}, t_{2}\right)=\frac{2}{3} C_{F} C_{A}^{2} \kappa_{1} \frac{g_{0}^{6}}{(4 \pi)^{4}} u^{4 \varepsilon} t_{1}^{2 \varepsilon} t_{2}^{2 \varepsilon}\left(t_{2}-t_{1}\right)^{-2+2 \varepsilon} \exp \left[-\frac{i}{4} C_{A} \kappa_{0} \frac{g_{0}^{2}}{4 \pi} \frac{t_{2}^{2 \varepsilon}-t_{1}^{2 \varepsilon}}{\varepsilon u^{1-2 \varepsilon}}\right] .
$$


Now we consider just a single Coulomb gluon exchange between $t_{1}$ and $t_{2}$ :

$$
K^{(1)}\left(t_{1}, t_{2}\right)=-\frac{i}{6} C_{F} C_{A}^{3} \kappa_{2} \frac{g_{0}^{8}}{(4 \pi)^{5}} \frac{t_{1}^{2 \varepsilon} t_{2}^{2 \varepsilon}\left(t_{2}^{2 \varepsilon}-t_{1}^{2 \varepsilon}\right)\left(t_{2}-t_{1}\right)^{-2+2 \varepsilon}}{\varepsilon u^{1-6 \varepsilon}} .
$$

Calculating the integral (4.3) by the substitutions $t_{1}=x t_{2}$ we obtain

$$
\int_{0}^{1} d x x^{2 \varepsilon}\left(1-x^{2 \varepsilon}\right)(1-x)^{-2+2 \varepsilon}=\frac{\Gamma(1+2 \varepsilon)}{1-2 \varepsilon}\left[3 \frac{\Gamma(1+4 \varepsilon)}{\Gamma(1+6 \varepsilon)}-2 \frac{\Gamma(1+2 \varepsilon)}{\Gamma(1+4 \varepsilon)}\right]=1+\mathscr{O}(\varepsilon),
$$

and

$$
R_{\mathrm{us}}^{(1)}=-\frac{i}{48} C_{F} C_{A}^{3} \kappa_{3} \frac{g_{0}^{8}}{(4 \pi)^{5}} \frac{T^{8 \varepsilon}}{\varepsilon^{2} u^{1-6 \varepsilon}} .
$$

The soft contribution is nearly local in time $\left(t_{2}-t_{1} \sim u t_{1,2} \ll t_{1,2}\right)$, and can be described by a soft potential. For a single Coulomb exchange between $t_{1}$ and $t_{2}$, it is

$$
V_{\mathrm{soft}}^{(1)}(r)=c C_{F} C_{A}^{3} \frac{g_{0}^{8}}{r^{1-8 \varepsilon}}
$$

by counting dimensions, so that

$$
R_{\mathrm{soft}}^{(1)}=-i \int_{0}^{T} d t V_{\mathrm{soft}}^{(1)}(u t)=-i c C_{F} C_{A}^{3} \frac{g_{0}^{8} T^{8 \varepsilon}}{8 \varepsilon u^{1-8 \varepsilon}}
$$

The double pole $1 / \varepsilon^{2}$ should cancel in $R^{(1)}=R_{\mathrm{us}}^{(1)}+R_{\mathrm{soft}}^{(1)}$; this fixes the $1 / \varepsilon$ term in $c$, and we obtain

$$
R^{(1)}=-\frac{i}{48} C_{F} C_{A}^{3} \frac{g_{0}^{8} T^{8 \varepsilon}}{(4 \pi)^{5}} \frac{\kappa_{3} u^{6 \varepsilon}-\kappa_{4} u^{8 \varepsilon}}{\varepsilon^{2} u}=\frac{i}{24} C_{F} C_{A}^{3} \frac{\alpha_{s}^{4}(\mu)(\mu T)^{8 \varepsilon}}{4 \pi} \frac{\log u+\text { const }}{\varepsilon u} .
$$

This leads to the following contribution to $\Gamma_{\text {cusp }}[17]$ :

$$
\Delta \Gamma_{\text {cusp }}=-\frac{i}{3} C_{F} C_{A}^{3} \frac{\alpha_{s}^{4}}{4 \pi} \frac{\log u+\text { const }}{u} .
$$

Finally, analytically continuing it to Euclidean space $\left(\varphi_{E}=\pi+i \varphi_{M}, \varphi_{M}=u\right)$, we obtain

$$
\Delta \Gamma_{\text {cusp }}(\pi-\delta)=-\frac{1}{3} C_{F} C_{A}^{3} \frac{\alpha_{s}^{4}}{4 \pi} \frac{\log \delta+\text { const }}{\delta} .
$$

Acknowledgements. AG thanks N. Brambilla and A. Vairo for discussions of $\Gamma_{\text {cusp }}(\pi-\delta)$ (Sect. 4). This work was supported in part by the Russian Ministry of science and higher education, by the research training group GRK Symmetry Breaking (DFG/GRK 1581), by the Deutsche Forschungsgemeinschaft through the project "Infrared and threshold effects in QCD", by a GFK fellowship and by the PRISMA cluster of excellence at JGU Mainz. The authors gratefully acknowledge the computing time granted on the supercomputer Mogon at JGU Mainz. 


\section{References}

[1] K. Melnikov and T. van Ritbergen, The three-loop on-shell renormalization of QCD and QED, Nucl. Phys. B 591 (2000) 515 [hep-ph/0005131].

[2] K. G. Chetyrkin and A. G. Grozin, Three-loop anomalous dimension of the heavy-light quark current in HQET, Nucl. Phys. B 666 (2003) 289 [hep-ph/0303113].

[3] A. Grozin, J. M. Henn, G. P. Korchemsky and P. Marquard, Three-loop cusp anomalous dimension in QCD, Phys. Rev. Lett. 114 (2015) no. 6, 062006 [arXiv:1409.0023 [hep-ph]].

[4] A. Grozin, J. M. Henn, G. P. Korchemsky and P. Marquard, The three-loop cusp anomalous dimension in QCD and its supersymmetric extensions, JHEP 01 (2016) 140 [arXiv:1510.07803 [hep-ph]].

[5] D. J. Broadhurst and A. G. Grozin, Matching QCD and HQET heavy-light currents at two loops and beyond, Phys. Rev. D 52 (1995) 4082 [hep-ph/9410240].

[6] M. Beneke and V. M. Braun, Power corrections and renormalons in Drell-Yan production, Nucl. Phys. B 454 (1995) 253 [hep-ph/9506452].

[7] A. Grozin, Leading and next-to-leading large- $n_{f}$ terms in the cusp anomalous dimension and quark-antiquark potential, PoS LL 2016 (2016) 053 [arXiv:1605.03886 [hep-ph]].

[8] P. Marquard, A. V. Smirnov, V. A. Smirnov and M. Steinhauser, Four-loop wave function renormalization in QCD and QED, Phys. Rev. D 97 (2018) no. 5, 054032 [arXiv:1801.08292 [hep-ph]].

[9] R. Brüser, A. G. Grozin, J. M. Henn, M. Stahlhofen, work in progress.

[10] J. M. Henn, A. V. Smirnov, V. A. Smirnov and M. Steinhauser, A planar four-loop form factor and cusp anomalous dimension in QCD, JHEP 05 (2016) 066 [arXiv:1604.03126 [hep-ph]].

[11] J. Davies, A. Vogt, B. Ruijl, T. Ueda and J. A. M. Vermaseren, Large- $n_{f}$ contributions to the four-loop splitting functions in QCD, Nucl. Phys. B 915 (2017) 335 [arXiv:1610.07477 [hep-ph]].

[12] A. Grozin, Four-loop cusp anomalous dimension in QED, JHEP 06 (2018) 073 [arXiv:1805.05050 [hep-ph]].

[13] A. Grozin, J. Henn and M. Stahlhofen, On the Casimir scaling violation in the cusp anomalous dimension at small angle, JHEP 10 (2017) 052 [arXiv:1708.01221 [hep-ph]].

[14] S. Moch, B. Ruijl, T. Ueda, J. A. M. Vermaseren and A. Vogt, Four-loop non-singlet splitting functions in the planar limit and beyond, JHEP 10 (2017) 041 [arXiv:1707.08315 [hep-ph]].

[15] S. Moch, B. Ruijl, T. Ueda, J. A. M. Vermaseren and A. Vogt, On quartic colour factors in splitting functions and the gluon cusp anomalous dimension, Phys. Lett. B 782 (2018) 627 [arXiv:1805.09638 [hep-ph]].

[16] J. Henn, R. N. Lee, A. V. Smirnov, V. A. Smirnov and M. Steinhauser, Four-loop photon quark form factor and cusp anomalous dimension in the large- $N_{c}$ limit of QCD, JHEP 03 (2017) 139 [arXiv:1612.04389 [hep-ph]].

[17] A. G. Grozin, M. Stahlhofen, work in progress.

[18] B. Ruijl, T. Ueda, J. A. M. Vermaseren and A. Vogt, Four-loop QCD propagators and vertices with one vanishing external momentum, JHEP 06 (2017) 040 [arXiv:1703.08532 [hep-ph]]. 
[19] C. Anzai, Y. Kiyo and Y. Sumino, Violation of Casimir scaling for static QCD potential at three-loop order, Nucl. Phys. B 838 (2010) 28, Erratum: Nucl. Phys. B 890 (2015) 569 [arXiv:1004.1562 [hep-ph]].

[20] R. H. Boels, T. Huber and G. Yang, Four-loop nonplanar cusp anomalous dimension in $\mathscr{N}=4$ supersymmetric Yang-Mills theory, Phys. Rev. Lett. 119 (2017) no.20, 201601 [arXiv:1705.03444 [hep-th]].

[21] R. H. Boels, T. Huber and G. Yang, The Sudakov form factor at four loops in maximal super Yang-Mills theory, JHEP 01 (2018) 153 [arXiv:1711.08449 [hep-th]].

[22] E. Panzer, Algorithms for the symbolic integration of hyperlogarithms with applications to Feynman integrals, Comput. Phys. Commun. 188 (2015) 148 [arXiv:1403.3385 [hep-th]].

[23] R. N. Lee, A. V. Smirnov, V. A. Smirnov and M. Steinhauser, Analytic three-loop static potential, Phys. Rev. D 94 (2016) no.5, 054029 [arXiv:1608.02603 [hep-ph]].

[24] W. Kilian, T. Mannel and T. Ohl, Unimagined imaginary parts in heavy quark effective field theory, Phys. Lett. B 304 (1993) 311 [hep-ph/9303224].

[25] N. Brambilla, A. Pineda, J. Soto and A. Vairo, The Infrared behavior of the static potential in perturbative QCD, Phys. Rev. D 60 (1999) 091502 [hep-ph/9903355];

[26] N. Brambilla, A. Pineda, J. Soto and A. Vairo, Potential NRQCD: An Effective theory for heavy quarkonium, Nucl. Phys. B 566 (2000) 275 [hep-ph/9907240]. 